\title{
Plasmonic-Resonant Bowtie Antenna for Carbon Nanotube Photodetectors
}

\author{
Hongzhi Chen, ${ }^{1}$ Ning Xi, ${ }^{1}$ King Wai Chiu Lai, ${ }^{1}$ Liangliang Chen, ${ }^{1}$ \\ Carmen Kar Man Fung, ${ }^{1}$ and Jianyong Lou $^{2}$ \\ ${ }^{1}$ Department of Electrical and Computer, Engineering, Michigan State University, East Lansing, MI 48824, USA \\ ${ }^{2}$ State Key Laboratory of Electrical Insulation and Power Equipment, Xi'an Jiaotong University, Xi'an 710049, China
}

Correspondence should be addressed to Hongzhi Chen, chenhon5@msu.edu

Received 12 September 2011; Accepted 17 November 2011

Academic Editor: A. Femius Koenderink

Copyright () 2012 Hongzhi Chen et al. This is an open access article distributed under the Creative Commons Attribution License, which permits unrestricted use, distribution, and reproduction in any medium, provided the original work is properly cited.

\begin{abstract}
The design of bowtie antennas for carbon nanotube (CNT) photodetectors has been investigated. CNT photodetectors have shown outstanding performance by using CNT as sensing element. However, detection wavelength is much larger than the diameter of the CNT, resulting in small fill factor. Bowtie antenna can confine light into a subwavelength volume based on plasmonic resonance, thus integrating a bowtie antenna to CNT photodetectors can highly improve photoresponse of the detectors. The electric field enhancement of bowtie antennas was calculated using the device geometry by considering fabrication difficulties and photodetector structure. It is shown that the electric field intensity enhancement increased exponentially with distance reduction between the CNT photodetector to the antenna. A redshift of the peak resonance wavelength is predicted due to the increase of tip angles of the bowtie antennas. Experimental results showed that photocurrent enhancement agreed well with theoretical calculations. Bowtie antennas may find wide applications in nanoscale photonic sensors.
\end{abstract}

\section{Introduction}

With the development of nanotechnologies, one-dimensional (1D) materials, including nanowires and nanotubes, emerged as important components in photonics and optoelectronics [1]. Compared to bulk materials, 1D materials possess a number of unique properties arose from their geometries, making them potential candidates to outperform traditional optical devices. In particular, carbon nanotubes (CNTs) are of great interest for exploring as functional elements for 1D photodetectors due to their perfect nanohollow cylinder structure [2, 3]. However, a critical challenge for obtaining high-performance 1D photodetectors is the dimension incompatibility between photodetectors and optical wavelengths. The radial sizes of CNTs are much smaller than detection wavelengths, resulting in low fill factor and responsivity. Motivated by this challenge, optical antennas were designed to integrate with CNT photodetectors so as to bridge the gap between nanosize sensing elements and microsize wavelengths. Due to plasmonic resonance, optical antennas can confine strong optical near fields in a volume far below a cubic wavelength of excitation light. This can greatly enhance photon absorption owing to the increase of coupling between CNT and vibrating electrical fields of photons. By integrating CNT photodetectors with optical antennas, the nanoscale photodetectors can be made with outstanding performance and photon absorption efficiency.

There are two main categories of photodetectors: thermal detectors and photonic detectors. Both thermal detectors and photonic detectors have their limitations: thermal detectors have slow response and low detectivity, while traditional photon detectors can only detect a narrow spectrum, which is limited by their bandgap energies. Some of the photon detectors need to operate with cryogenic cooling system, particularly middle-wave infrared (IR) or longer wavelength detectors. Photodetectors using nanomaterials as core materials were proposed to overcome these problems, since nanomaterials, especially CNTs, have a number of exceptional optoelectronic properties [4]. CNT-based photodetectors were extensively investigated. Multiexiton was observed in a CNT photodetector, showing the potential of making photodetectors with higher responsivity [5]. A photovoltaic 
CNT detector was reported to detect middle-wave IR at room temperature [6]. A spectral tunable CNT photodetector could modulate its detection spectrum by electrically tailoring the diameters of CNTs [7], because the bandgap energies of the 1D materials are inversely proportional to their diameters [8]. All these promising properties stem from the $1 \mathrm{D}$ quantum confined geometry of CNTs, which, nevertheless, cause low fill factor and absorption. In order to fully employ the exceptional advantages of the CNTs in photosensors, the problem of low absorption needs to be addressed, and optical antennas are promising in solving this issue.

In our design, the optical antenna performs as a plasmonic lens that funnels light into the CNT photodetector. This is analogous to the microlenses on top of conventional charge coupled device (CCD) array to increase detector fill factor $[9,10]$. If the CNT photodetector occupied the same area as the antenna/CNT photodetector, they have same fill factor. However, technical difficulties prevent the assembling of a large array of uniform CNTs. Assembling a CNT thin film has been demonstrated, but the interaction between CNTs in thin film resulted in the loss of their unique optical properties. Therefore, one single CNT is more favorable and easier to manipulate in order to fabricate high-performance photodetectors. But the challenge of one individual CNT as the core material for photodetectors is the small diameter of CNT. The optical antenna can strongly concentrate light into subwavelength volume that couples to the CNT in order to improve fill factor.

Optical antennas have invoked considerable interests, because of their ability to generate large and localized electromagnetic fields. The basic principle of optical antennas is based on the resonant behavior between excitation light and metal structure. When light interacts with optical antennas that have proper dimensions, surface plasmons are generated due to resonant coupling between the electromagnetic surface waves and oscillations of free electrons in the metal. Light irradiating on the optical antenna at frequencies below the resonant frequency is reflected, since it induces motion in the charge carriers that act to screen out the incident field; when the frequency of light is above the resonant frequency, light is transmitted because the charges are unable to respond quickly enough to screen out the incident field [11]; only at the resonant frequency, light is resonant coupling to free charges of metals, and the incident waves will be absorbed and reradiated. By properly orienting the reradiated waves, intensely localized near-fields can be generated. Confining or localizing light into a small volume has found plenty of applications in many research fields, including spectroscopy, microscopy, lithography, biosensing, chemical studies, and so on $[12,13]$. In recent years, optical antennas used to improve the performance of planar photovoltaic devices became a hot field [14]. However, very little research was conducted to improve the performance of $1 \mathrm{D}$ photodetectors using optical antenna, although 1D materials become an emerging platform in photovoltaic devices [15].

There are a variety of novel shaped optical antennas, such as nanosphere, nanorod and nanotriangle monomers, nanosphere dimmers, dipole antennas, bowtie antennas,
Yagi-Uda antennas, cross-antennas, and so on [13]. Among all these optical antennas, dipole and bowtie antennas are particularly suitable for integrating with $1 \mathrm{D}$ photodetectors because of their geometries. Dipole antennas are nanorod dimmers, while bowtie antennas are nanotriangle dimmers, therefore, both dipole and bowtie antennas can be considered as two opposing tip-to-tip nanotriangles (nonzero apex width) with tip angles ranging from $0^{\circ}$ (dipole antennas) to wider angles. The coupling between two pieces of metals in these antennas concentrates the incident light into a nanoscale gap, which locates above a CNT photodetector in order to enhance the interaction between the localized nearfields and CNTs.

There were a number of approaches to improve a photodetector's performance using plasmonic resonance: photocurrent enhancement was demonstrated in an Si photodiode via the excitation of plasmon resonances in spherical Au nanoparticles deposited on the Si surface [16]; a middlewave IR photodetector, comprised of a metallic grating filled with $\mathrm{HgCdTe}$, was proposed to improve the signalto-noise ratio by plasmonic resonance [17]; an optical antenna was utilized as a metal electrode for a Si Schottky diode to boost photo-detection [18]. Optical antennas were embedded in these photodetectors, acting as part of the photodetectors. In our design, the bowtie antenna performs as a plasmonic lens, which can be easily integrated into all types of high performance 1D photodetectors [3, 19-22]. In particular, the bowtie antenna perfectly matches with the CNT photodetector due to the compatibility of the diameter of CNT and gap distance of the bowtie antenna, because the electric field ( $E$-field) enhancement exponentially increases with decreasing gap distance [23].

The properties of free-standing optical bowtie antennas have been studied extensively [23, 24], and our group has demonstrated that dipole antenna can boost photoresponse of 1D IR detectors [25]. However, the design of bowtie antennas for 1D photodetectors was not systematically studied. In this paper, we will present the design of bowtie antennas that can be integrated with CNT photodetectors to highly improve the detection of IR signal with wavelength of $830 \mathrm{~nm}$. E-field enhancement with bowtie antennas was calculated to seek the optimized tip angle and insulator thickness. Experimental results showed that the photocurrent enhancement was 12.67 times by adding a bowtie antenna with precise alignment and dimension control in fabrication. Calculation of relative energy difference on the CNT photodetector at absence and presence of the bowtie antenna quantitatively explained the photocurrent enhancement.

\section{Design of Optical Antennas}

Before the discussion of antenna design, an introduction of CNT photodetectors will be provided. The basic structure of a CNT photodetector is sketched in Figure 1(a), comprised of a single CNT connecting to two electrodes with high/low workfunctions ( $\mathrm{Ti}$ and $\mathrm{Au}$ ); the band diagram of the device is shown in Figure 1(b). On the left contact between Ti and CNT, Ti has a workfunction that is smaller than that of the CNT and forms a Schottky barrier; on the right contact, 


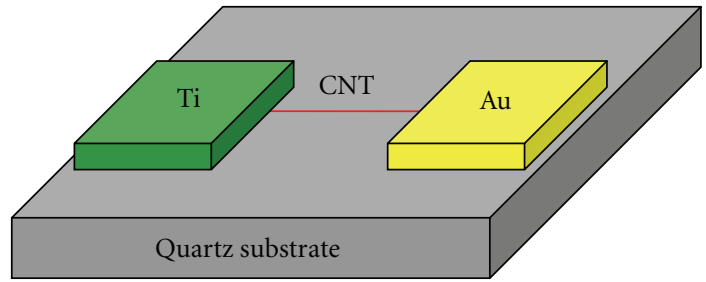

(a)

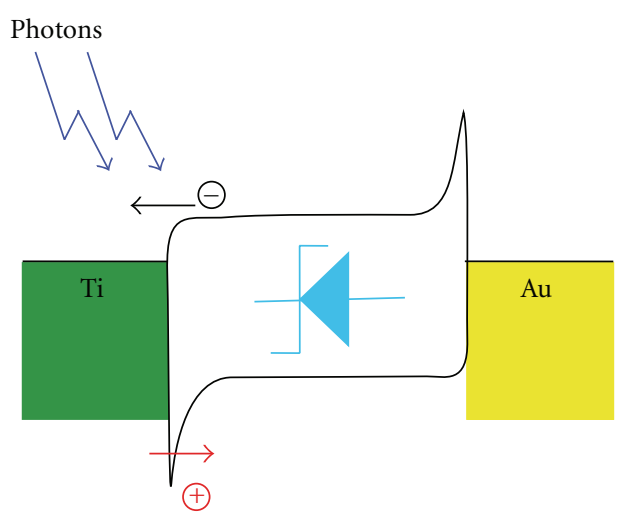

(b)

Figure 1: (a) The basic structure of a CNT photodetector, consisting of a CNT connecting to Ti and Au electrodes on top of a quartz substrate. (b) The band diagram of the CNT photodetector.

$\mathrm{Au}$ has a workfunction that is higher than (or similar with) that of the CNT and forms an ohmic contact (or quasiohmic contact) [22]. When the photons with energy higher than the CNT bandgap strike the CNT, electron and hole pairs will be generated, and be separated by the built-in potential of the Schottky barrier. We have demonstrated that CNT photodetectors exhibited outstanding performance to detect IR signals, and the sensitivity of photodetectors with asymmetric metal structure was much better than the ones with symmetric metal structures [22]. One thing should be noted is that the depletion region of the CNT photodetector may extend into the whole tube of the CNT, since the charge distribution of the 1D systems has a long-range tail [26], extending over the entire CNT [27]. In other words, the entire CNT can be considered as depletion region. Therefore, the optical antennas cannot only be located on the contacts, but they also can be placed on top of the CNT body.

The bowtie antenna consists of two tip-to-tip nanotriangles that can confine strong electromagnetic field between two triangles at resonance frequency. Light illuminating on the bowtie antennas will generate instantaneous current, which oscillates at the frequency along the polarization of the incident light. The instantaneous current is maximum near the center of the nanotriangle, and decreases toward the edges of the antenna. This leads to a high density of surface charge at the tip of the nanotriangles. Displacement current flows into the small air gap of the bowtie antennas, reradiating the absorbed energy. The strong coupling of these two opposing tip-to-tip triangles brings the center of the reradiating source to the center of the gap [28]. The maximum intensity enhancement can be obtained at resonance frequency, which is mainly determined by the dimension and materials of antennas.

The relative position of the CNT photodetector and bowtie antenna is shown in Figure 2. A thin insulator $\left(\mathrm{Si}_{3} \mathrm{~N}_{4}\right)$ is sandwiched between the bowtie antenna and the photodetector (Figure 2(c)), with the gap of bowtie antenna aligning to the center of CNT channel. The geometrical parameters are also denoted in Figure 2: where $d$ is the gap distance, $w$ is the apex width, $L$ is the length of the bowtie antenna, $\theta$ is the tip angle, and $t$ is the thickness of the insulator between antenna and CNT.

This CNT photodetector is designed for detecting IR signal at $830 \mathrm{~nm}$, thus the bowtie antenna should resonate at or close to this wavelength. Resonance wavelength is very sensitive to the dimensions of bowtie antennas, but several parameters are fixed in simulation considering the fabrication constraints, and some parameters have been extensively studied $[28,29]$ : the gap distance $d$ is set to be $30 \mathrm{~nm}$ for easier alignment of the bowtie antenna to the photodetector; apex width is $30 \mathrm{~nm}$ to leave some tolerance for electron beam lithography (EBL); antenna length $L$ is $400 \mathrm{~nm}$ because it is close to half-wavelength of the IR signal; the bowtie antenna is made of $3 \mathrm{~nm} \mathrm{Ti}$ and $20 \mathrm{~nm} \mathrm{Au}$. The width of both $\mathrm{Au}$ and $\mathrm{Ti}$ electrodes is $400 \mathrm{~nm}$, and the gap between two electrodes is $1000 \mathrm{~nm}$. The following simulation was based on the geometry including both electrodes and bowtie antennas, as shown in Figure 2.

The E-field enhancement spectra of bowtie antennas will be simulated with varying insulator thickness $t$ and tip angle $\theta$, not only since these two parameters can be easily tuned in real device fabrication, but they also have significant impacts to the E-field intensity enhancement.

Figure 2(a) also shows the direction of incident light polarization and the coordinate system for all numerical simulations using ANSYS HFSS: a plane wave is normally incident on the structure from the top with the electric field being polarized along the $x$ axis, which is parallel to the axis between two triangles, with electric field component amplitudes of $E_{x}=1 \mathrm{~V} / \mathrm{m}, E_{y}=E_{z}=0$.

\subsection{Insulator-Thickness-Dependent E-Field Enhancement.} The plasmonic resonance of the bowtie antennas was first investigated by varying the insulator thickness $t$ between the antenna to the CNT photodetector. This is a critical parameter, since the photodetector needs to be placed in close proximity to the optical antennas in order to fully take advantage of near fields [30]. The insulator-thicknessdependent E-field distribution at the substrate plane (the plane between $\mathrm{Si}_{3} \mathrm{~N}_{4}$ and quartz substrate), where CNT 


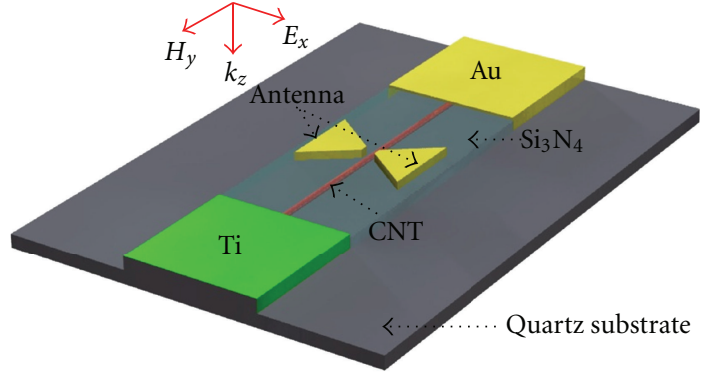

(a)

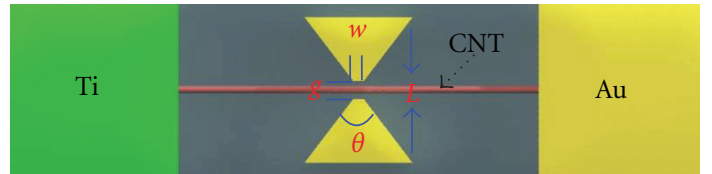

(b)

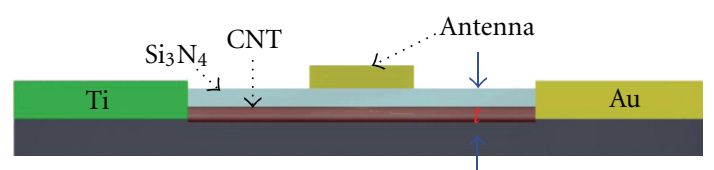

(c)

FIgURE 2: (a) A 3D geometry shows the relative position between the CNT photodetector and the bowtie antenna. The incident light with electric field polarized in the $x$ direction propagates in the $z$ direction. (b) Top view of the device, and parameters of the bowtie antenna: $L=400 \mathrm{~nm}$ is the length of the antenna, $g=30 \mathrm{~nm}$ is the gap of two pieces of the antenna, $w=30 \mathrm{~nm}$ is the apex width, and $\theta$ is the tip angle. (c) Side view of the device, showing the CNT photodetector and bowtie antenna sandwiching the $\mathrm{Si}_{3} \mathrm{~N}_{4}$ layer that has a thickness of $t$.

located, was calculated based on equilateral Au triangles $(\theta=$ $60^{\circ}$ ) with $d=30 \mathrm{~nm}, w=30 \mathrm{~nm}, L=400 \mathrm{~nm}$. The CNT photodetector and optical antenna are on the top of a quartz substrate, with a plane-wave light polarized in the $x$ direction normally illuminating the device from the top.

The resonance wavelength for this antenna is at $845 \mathrm{~nm}$ with $t=10 \mathrm{~nm}$. Figure 3 (a) shows the $E$-field distribution between two electrodes at the substrate plane for the device with $t=10 \mathrm{~nm}$ at its resonance wavelength. The $E$-field is confined into the gap of the bowtie antenna with the maximum amplitude located at the center of the gap due to the strong coupling between two nanotriangles. The Efield distribution is in the shape of a horizontal bowtie, and the amplitude of the $E$-field decreases as the distance to the center of the gap increases in the horizontal direction. The illuminating light had $E=1 \mathrm{~V} / \mathrm{m}$, while the peak amplitude at substrate plane became $E=14.608 \mathrm{~V} / \mathrm{m}$ at resonance after adding the bowtie antenna, with an enhancement of 14.608 fold.

The peak E-field intensity enhancement $\max \left(|E|^{2}\right)$ as a function of insulator thickness at resonance wavelength was shown in Figure 3(b). The E-field distributions with thickness of $\mathrm{Si}_{3} \mathrm{~N}_{4}$ ranging from $10 \mathrm{~nm}$ to $50 \mathrm{~nm}$ were investigated. The blue curve shows the peak intensity enhancement at the substrate plane: the enhancement decreased from 213.1 to 26.7 when the thickness of the $\mathrm{Si}_{3} \mathrm{~N}_{4}$ increased from $10 \mathrm{~nm}$ to $50 \mathrm{~nm}$. The red curve in Figure 3(b) is the exponential fit of the data, which matches very well with the blue curve. The resonance wavelength of the antenna redshifted from $845 \mathrm{~nm}$ to $1034 \mathrm{~nm}$ with the thickness increasing of $\mathrm{Si}_{3} \mathrm{~N}_{4}$ layer from $t=10 \mathrm{~nm}$ to $t=50 \mathrm{~nm}$ [31]. The inset of Figure 3(b) shows that the E-field enhancement at wavelength of $830 \mathrm{~nm}$, which corresponds to the wavelength of our laser source, decreases dramatically by increasing the insulator thickness. The simulation results indicate that the field intensity enhancement decreases significantly with the increasing thickness of the insulator, because evanescent waves decay exponentially from the antenna. Therefore, in order to fully use the power of the antenna, the photodetector needs to be fabricated close to the bowtie antenna.

2.2. Tip-Angle-Dependent E-Field Enhancement Spectra. Not only is the tip angle of the bowtie antennas easily modified in real device fabrication, but it also has critical impact on both resonance wavelength and $E$-field intensity enhancement. The E-field spectra as a function of the tip angle were calculated with $d=30 \mathrm{~nm}, w=30 \mathrm{~nm}, L=400 \mathrm{~nm}$, and $t=10 \mathrm{~nm}$. Figure 4 shows the $E$-field spectra of bowtie antenna with tip angles of 0 degrees (dipole antenna), 30 degrees, 60 degrees, 90 degrees, and 120 degrees.

The spectra vary significantly with different tip angles. It is shown that bowtie antennas cover wider spectrum with larger tip angles. Bowtie antennas can be considered as a two-dimensional analogue of biconical antenna, therefore, wider tip angles are expected to possess broader bandwidth. The bowtie antenna with tip angle of 120 degrees shows field intensity enhancement in a broadest spectrum. What is more, two resonance wavelengths was found with a fundamental resonance wavelength at $1.13 \mu \mathrm{m}$ and a highorder resonance at $0.77 \mu \mathrm{m}$. High-order resonance is easier to be excited for large angle Bowtie antennas, since larger angle bowtie antennas are prone to overcome the symmetry limitation of octupolar resonance, while octupolar resonance is forbidden in the 0 degree bowtie antenna under normal incidence [32].

The peak $E$-field intensity enhancement increases with wider tip angles when the tip angle is smaller than 60 degrees, while it reduces when the tip angle is larger than 90 degrees. In addition, the resonance wavelengths, where the peak intensity enhancements are obtained, redshift with 

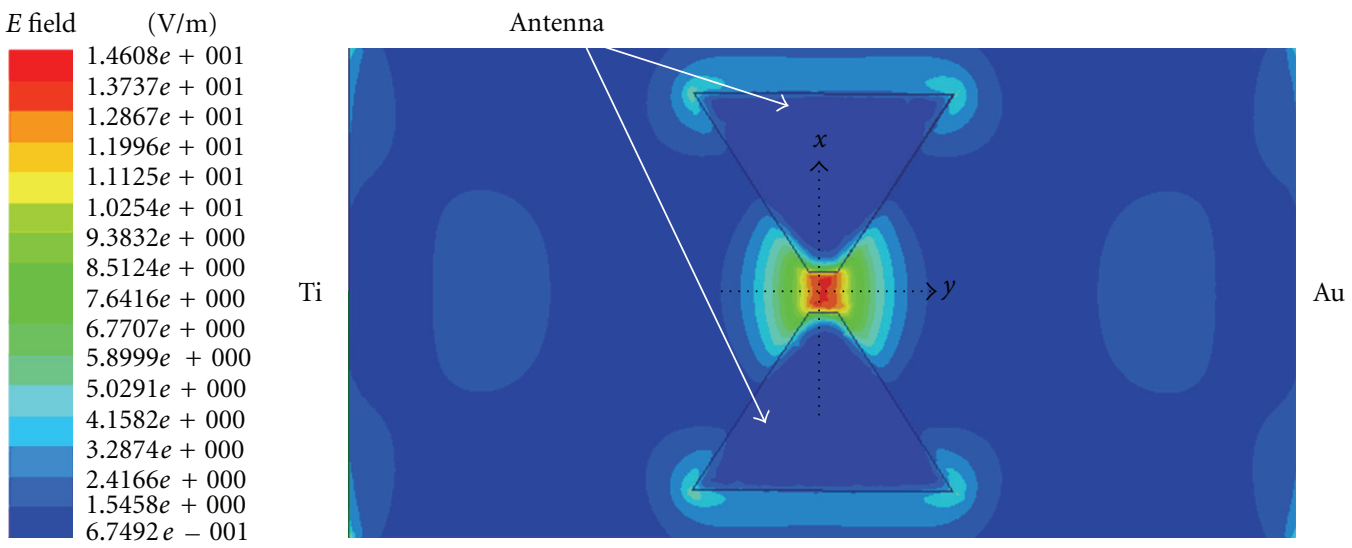

(a)

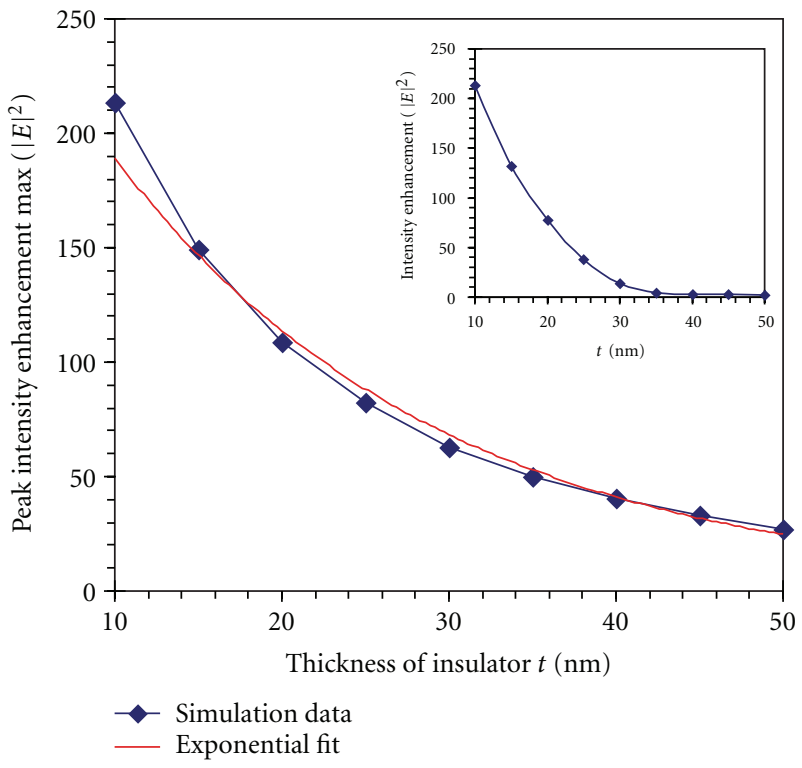

(b)

Figure 3: (a) The $E$-field distribution of the resonance frequency $(845 \mathrm{~nm})$ of the bowtie antenna with $\theta=60^{\circ}$, and $t=10 \mathrm{~nm}$. The $x-y$ axis show the coordinate for simulation. (b) The $t$ dependent peak field intensity max $\left(|E|^{2}\right)$, blue curve is the simulation values, and red curve is the exponential fit. The inset shows field intensity enhancement $|E|^{2}$ at wavelength of $830 \mathrm{~nm}$.

increasing tip angles. Resonance wavelength is proportional to the length of the triangle edges of bowtie antennas, and thus resonance wavelength redshifts as tip angle increases [33]. This can be explained as follows: the two opposite $\mathrm{Au} /$ air interfaces in one cross-section vertical to the antenna axis are far away from each other in the $y$ direction, thus the local surface plasmon polariton is confined at one Au/air interface and not affected by the opposite one, especially when the cross-section is close to the extremity edges. As a result, the bowtie antennas are dominated by isolated surface plasmon polaritons that propagate along the triangle edges; consequently, a standing wave along the triangle edges is generated, with resonance wavelength scales with the length of the triangle edges, therefore, redshifts as the tip angle increases [32]. The length of triangle edges of the bowties increases from $185 \mathrm{~nm}$ to $272 \mathrm{~nm}$ when the tip angle is widened from 0 degrees to 90 degrees, with an increment of only $87 \mathrm{~nm}$. As a result, the cross-section along $y$ axis from the tip to extremity edges changes gradually, and thus resonance wavelength differs slightly. The length of triangle edge increases to $383 \mathrm{~nm}$ when the tip angle becomes 120 degrees. This major length change causes a rapid change of local cross-section, resulting in a big shift of the resonance wavelength.

The simulation results show that larger angle bowtie antennas can cover broader spectrum together with multiple resonances, which are suitable for photodetectors with widespectrum detection. In our experiment, we were detecting IR signals with single wavelength of $830 \mathrm{~nm}$. Bowtie antennas with a 60-degree tip angle were fabricated to verify the field 


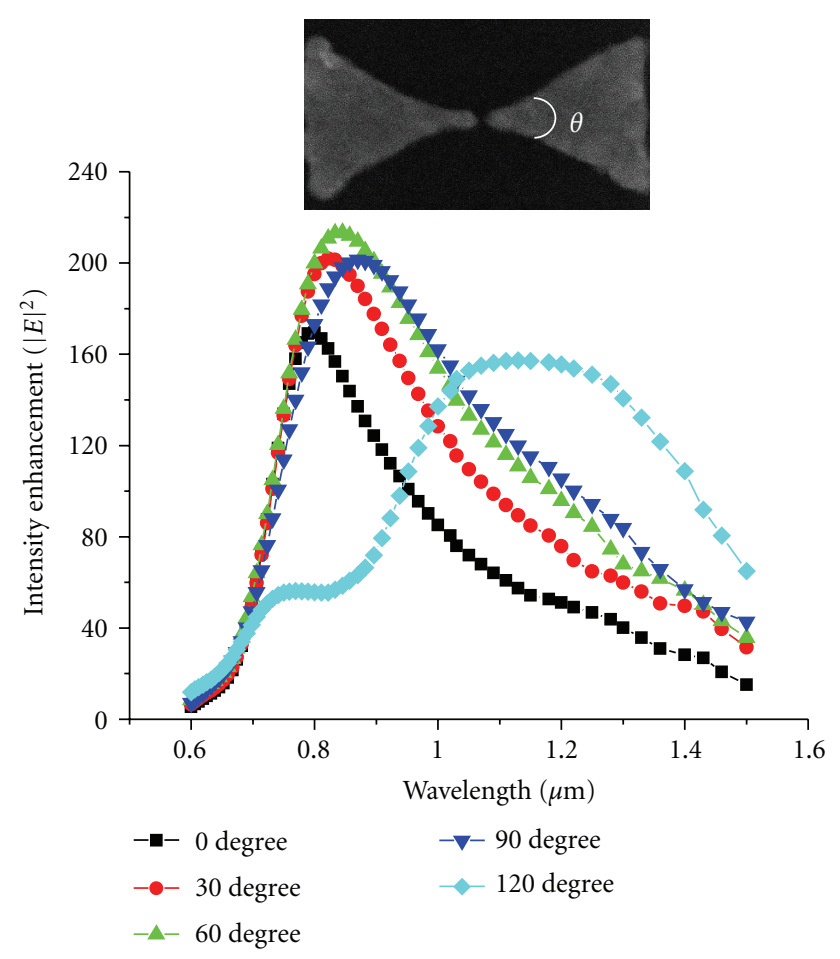

FIgURE 4: E-field intensity enhancement $|E|^{2}$ spectra of bowtie antennas with tip angles ranging from 0 degrees to 120 degrees. The inset shows a scanning electron microscopy (SEM) image of a bowtie antenna, denoting the tip angle.

intensity enhancement of the antennas, since the highest peak field intensity enhancement is obtained at this tip angle, and whose resonance wavelength is close to $830 \mathrm{~nm}$.

\section{Fabrication and Experimental Results}

In order to investigate the performance of bowtie antennas, $\mathrm{Au}$ bowties were fabricated on top of a CNT photodetector as depicted in Figure 2(a). Photocurrent of CNT photodetector with absence and presence of bowtie antennas was compared so as to examine the field intensity enhancement of the antennas.

3.1. Fabrication Process. The fabrication process starts with EBL an Au electrode on top of a quartz substrate. Prior to EBL, the quartz substrate was cleaned in acetone and bombarded with oxygen plasma for 5 minutes to remove residues. A $360 \mathrm{~nm}$ thick layer of polymethyl-methacrylate copolymer resist (PMMA/MMA) (9\% in Ethyl Lactate, from MicroChem Corp.) was spun at $4000 \mathrm{rpm}$ on the substrate, and the sample was baked in a hot plate at $175^{\circ} \mathrm{C}$ for 5 minutes to harden the resist and remove solvent. It was followed by spinning a layer of polymethyl-methacrylate (PMMA) (2\% in Chlorobenzene, from MicroChem Corp.) at $4000 \mathrm{rpm}$ with a thickness of $130 \mathrm{~nm}$ to form a bilayer resist. After that, the sample was baked in a hot plate at $175^{\circ} \mathrm{C}$ for 30 minutes to remove solvent residue. A thin layer of $\mathrm{Au}$ with thickness of $10 \mathrm{~nm}$ was deposited on top of the resist to avoid charging effect on the sample during EBL writing. The resist was exposed with the patterned of an $\mathrm{Au}$ electrode with width of $400 \mathrm{~nm}$ and some alignment mark at an acceleration voltage of $30 \mathrm{kV}$ and an area dose of $290 \mu \mathrm{C} / \mathrm{cm}^{2}$. After exposure, the thin Au layer on the resist was stripped using gold etching for 10 seconds, and then the resist was developed using a 1:3 methyl-iso-butylketone (MIBK) in isopropyl alcohol (IPA) solution for 29 seconds. Following development, the sample was rinsed with DI water, and bombarded by oxygen plasma for 90 seconds to remove residual resist. A $3 \mathrm{~nm}$ thick Ti adhesion layer and $40 \mathrm{~nm}$ thick Au layer were deposited using a thermal evaporator. The $\mathrm{Au}$ electrode and alignment mark were transferred to the substrate via a lift-off process using acetone (Figure 5(a)). Following the same processes with precise alignment, another electrode made by $\mathrm{Ti}$ was fabricated on the quartz substrate, with a gap of $1 \mu \mathrm{m}$ to the Au electrode (Figure 5(b)). A droplet of CNT suspension in ethanol alcohol was dispersed at the vicinity to the gap of electrodes, and an $\mathrm{AC}$ voltage of $1 \mathrm{~V}_{\mathrm{pp}}$ and $10 \mathrm{kHz}$ frequency was applied between the electrodes through the dielectrophoresis (DEP) deposition system [34]. With the assistance of an atomic force microscope (AFM) manipulation system [35], an individual single wall CNT (SWCNT), with diameter of 1-2 nm, was assembled between the electrodes, as shown in the inset of Figure 5(c), which is the AFM image of the CNT photodetector. A detailed deposition process was introduced in [36]. After that, a layer of $\mathrm{Si}_{3} \mathrm{~N}_{4}$ with thickness of $10 \mathrm{~nm}$ was deposited on top of the device using plasmaenhanced chemical vapor deposition (PECVD). The PECVD is a uniform deposition process, but Figure 5(d) shows the $\mathrm{Si}_{3} \mathrm{~N}_{4}$ only in the region between two electrodes for making the schematic clearer. Eventually, a bowtie antenna with two tip-to-tip equilateral $\mathrm{Au}$ triangles was fabricated on top of the $\mathrm{Si}_{3} \mathrm{~N}_{4}$ layer by aligning the gap of bowtie antenna to the SWCNT between two electrodes. The fabrication of the bowtie antenna started with spinning a $100 \mathrm{~nm}$ thick layer of PMMA at $6000 \mathrm{rpm}$ on the device, and the sample was baked on a hot plate at $175^{\circ} \mathrm{C}$ for 30 minutes. After that, an Au layer of $10 \mathrm{~nm}$ was deposited on top of the device to prevent charging effect. In order to precisely align the antenna to the detector, AFM was used to record the position of the SWCNT for designing antenna layout. The bowtie antenna was layout using single pass lines to reduce proximity effects in order to increase resolution. During EBL, the resist was exposed with a line dose of $0.75 \mu \mathrm{C} / \mathrm{cm}$ to form the pattern of bowtie antenna. After EBL, the thin Au layer was stripped, and the resist was developed using a 1:3 MIBK in IPA solution for 29 seconds. Following development, a $2 \mathrm{~nm}$ thick $\mathrm{Ti}$ adhesion layer and $20 \mathrm{~nm}$ Au layer were then deposited using a thermal evaporator. The antenna pattern was transferred to the substrate via a lift-off process Figure 5(e).

Figure 5(f) shows a 3D AFM image of one of the devices integrating bowtie antenna to a CNT photodetector. The SWCNT was not located at the center of the axis of two electrodes, thus the position of bowtie antenna was adjusted according to the position of the CNT. AFM images cannot show accurate dimensions in $x-y$ plane due to the image broadening, which is caused by the large radius of 


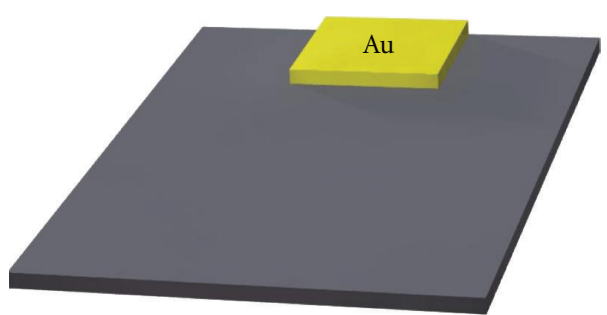

(a)

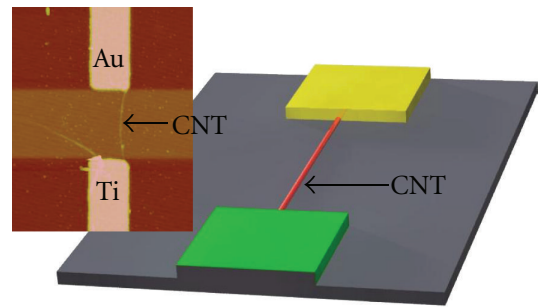

(c)

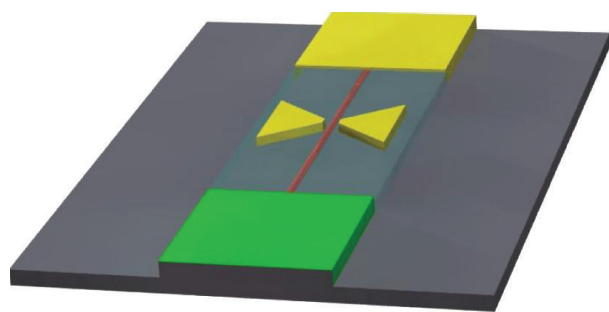

(e)

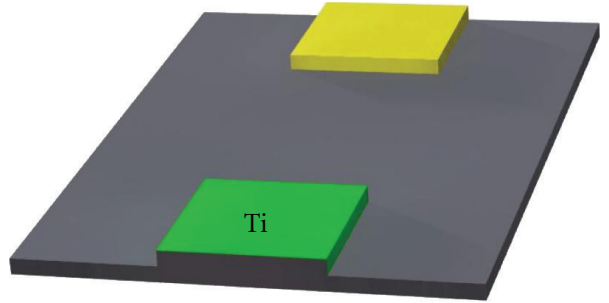

(b)

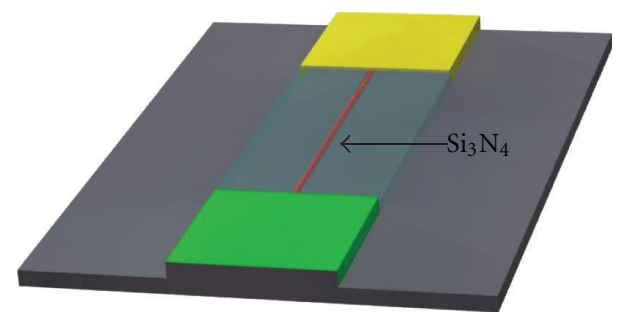

(d)

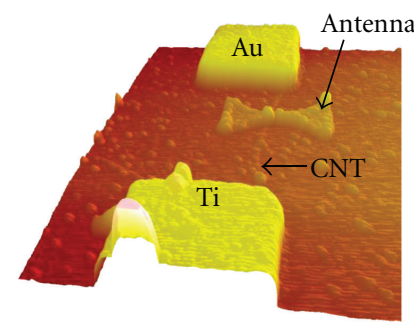

(f)

FIgURE 5: (a-e) Fabrication process of integrating a bowtie antenna to a CNT photodetector. The inset of (c) is an AFM image of an SWCNT bridging a Ti and an Au electrode. (f) A 3D AFM image of the device.

curvature of the apex of AFM tips, therefore, two triangles of the bowties in the AFM image seems connecting. SEM was utilized to measure the real dimensions of the bowtie antennas. The gap of bowtie antennas $(g)$ was $30 \pm 10 \mathrm{~nm}$, the apex width $(w)$ of the triangles was $30 \pm 10 \mathrm{~nm}$, length of the bowtie antenna $(L)$ was $400 \pm 30 \mathrm{~nm}$.

3.2. Experimental Results. Photocurrent measurements were carried out on CNT photodetectors with absence and presence of bowtie antennas so as to investigate the field enhancement of the antennas. An IR laser, which had $830 \mathrm{~nm}$ wavelength and polarization in $x$ direction (the axis of bowtie antenna), illuminated on the CNT photodetector that was housed in a testing chamber, and electrical signals were measured using Agilent semiconductor analyzer 4156C.

Photoresponse of the CNT photodetector without bowtie antenna was firstly recorded at room temperature by switching the IR laser on and off at $0.2 \mathrm{~Hz}$ for several cycles (blue curve of Figure 6). Photoresponse of the CNT photodetector was measured again after the fabrication of the bowtie antenna (red curve of Figure 6). The photocurrent is the current difference at presence and absence of IR illumination. The comparison of photoresponses was based

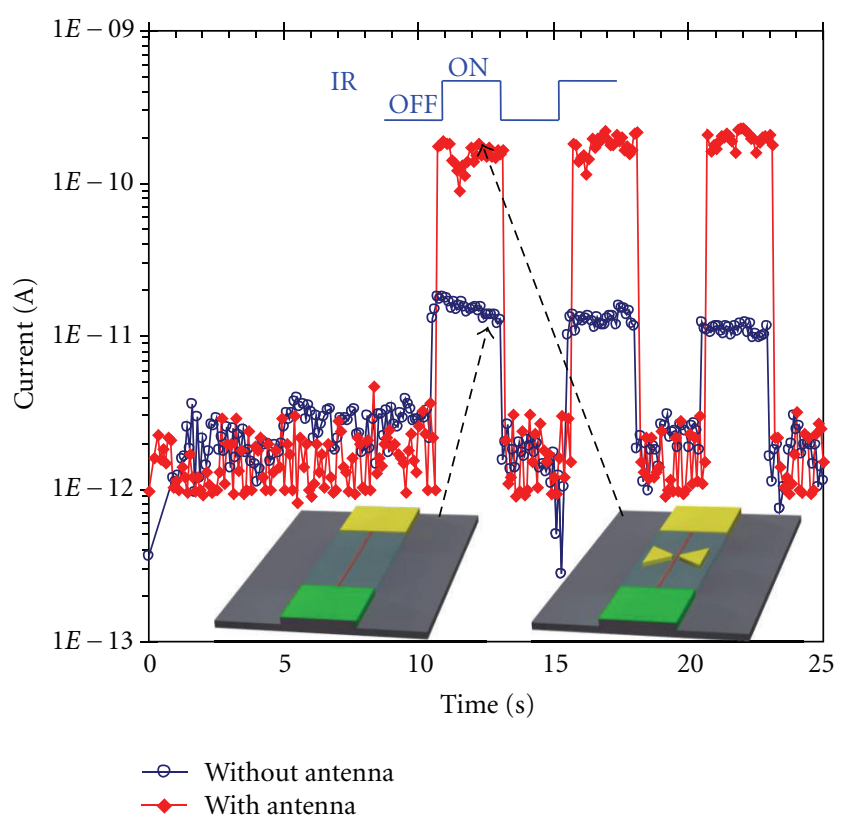

FIGURE 6: Photoresponses of the CNT photodetector without and with bowtie antenna by switching IR laser on and off at $0.2 \mathrm{~Hz}$. 


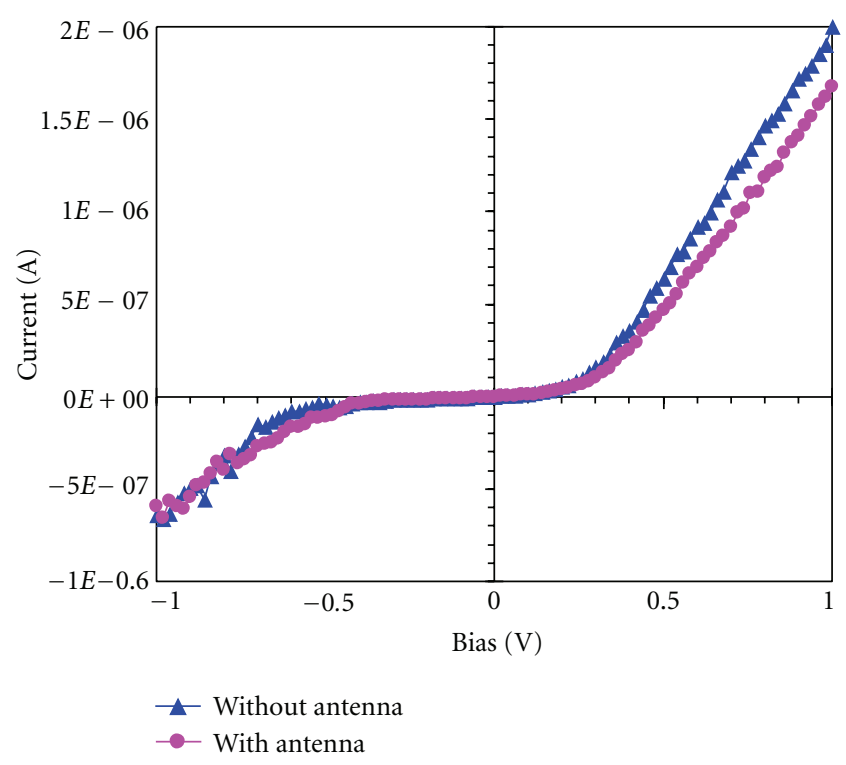

FIGURE 7: I-V characteristics of the CNT photodetector without and with the bowtie antenna.

on the amplitude change of photocurrent. Figure 6 shows that the dark current was $\sim 2 \times 10^{-12}$ A at the absence of IR illumination, and the current was increased significantly upon light illumination because of the photons generated electron and hole pairs within the CNT, which were separated in the Schottky barrier to produce photocurrent. The current dropped to the dark current when the IR laser was turned off. The reproducibility of the photoresponse was excellent. The photocurrent for CNT photodetector without antenna was $\sim 1.5 \times 10^{-11} \mathrm{~A}$, and it was increased to $\sim 1.9$ $\times 10^{-10} \mathrm{~A}$ after the fabrication of the bowtie antenna, with a photocurrent enhancement of 12.67 times.

The bowtie antenna functions as a plasmonic lens to funnel light into the CNT, which has a size much smaller than the gap of bowtie antenna. Hence, the area of detector material (physical cross-section) and absorption probability did not change with the absence or presence of the antenna. With the presence of bowtie antenna, the photon energy density on the detector material was enhanced due to subwavelength near-field confinement. It has been defined that the absorption cross-section of the detector is the total light power absorbed by detector material normalized by the incident photon flux [17]. Therefore, calculation of relative energy intensity difference on the CNT body at absence and presence of antenna will directly reflect the photocurrent enhancement.

The field intensity increment on the CNT can be calculated by integrating the E-field intensity along the CNT by $I=\int|E(y)|^{2} d y$ (Figure 3(a)), since the depletion region of the asymmetric CNT photodetector extended to the whole CNT. In other words, the photocurrent enhancement was caused by the E-field intensity increment on the CNT. Without the nanoantenna, the total field intensity on the CNT is equal to $10^{-6} \mathrm{~V}$ because $E_{x}(y)=1 \mathrm{~V} / \mathrm{m}$ and $y=$ $1 \mu \mathrm{m}$. The field intensity distribution with the antenna was calculated by using the parameters that were the same as fabricated device and experimental setup $(d=30 \mathrm{~nm}, w=$ $30 \mathrm{~nm}, L=400 \mathrm{~nm}, t=10 \mathrm{~nm}, \theta=60^{\circ}$, and radiation wavelength $=830 \mathrm{~nm}$ ). By integrating the field intensity along the $y$ axis between two electrodes (where the CNT located in Figure 3(a)), the field intensity was enhanced to $14.87 \times 10^{-6} \mathrm{~V}$, with a field enhancement of 14.87 fold. Therefore, the photocurrent enhancement is in good agreement with the simulation. Differences are attributed to geometry differences between the actual antennas and the simulation model, and some losses were occurred in the antennas due to edge and surface roughness.

In order to validate that the photocurrent enhancement was caused by the light confinement of the nanoantenna, rather than the contact condition change during fabrication, I-V characteristics of the photodetector without and with antenna were measured and shown in Figure 7. The I$\mathrm{V}$ characteristics showed current rectifying at low voltage, implying the existence of Schottky barriers. In addition, the bias-dependent currents of the CNT photodetector with absence and presence of nanoantenna were almost identical, which indicated that the contact condition did not change with the fabrication of the nanoantenna, since electrical signal was extremely sensitive to contact condition. This verifies that the photocurrent increment was caused by field intensity enhancement from the bowtie antenna.

\section{Conclusion}

The design of bowtie antennas integrating to the CNT photodetectors was studied. The E-field intensity enhancement of bowtie antennas with a small gap located on top of the asymmetric CNT photodetector was calculated. It was shown that the peak field intensity of the antenna was exponentially decreased with the distance between the antenna to the CNT photodetector. The resonance wavelength of the bowtie antenna redshifts with increasing tip angles. A fabrication process was developed to integrate the bowtie antenna with the CNT detector. It should be noted that the $1 \mathrm{D} \mathrm{CNT}$ detector is perfectly matched with the bowtie antenna, since the diameter of CNTs is in the same scale as the gap of the bowtie antenna. The experimental results agreed well with the simulation by measuring photocurrent enhancement. The nanoantenna may become an important component to the nanoscale photonic devices.

\section{Acknowledgement}

This research work is partially supported under NSF grant IIS 0713346, ONR Grants N00014-07-1-0935, and ONR Grants N00014-04-1-0799.

\section{References}

[1] R. Agarwal and C. M. Lieber, "Semiconductor nanowires: optics and optoelectronics," Applied Physics A, vol. 85, no. 3, pp. 209-215, 2006. 
[2] M. Freitag, Y. Martin, J. A. Misewich, R. Martel, and P. Avouris, "Photoconductivity of single carbon nanotubes," Nano Letters, vol. 3, no. 8, pp. 1067-1071, 2003.

[3] J. B. Zhang et al., "Photovoltaic effect in single carbon nanotube-based Schottky diodes," International Journal of Nanoparticles, vol. 6, no. 8, p. 11, 2008.

[4] P. Avouris, M. Freitag, and V. Perebeinos, "Carbon-nanotube photonics and optoelectronics," Nature Photonics, vol. 2, no. 6, pp. 341-350, 2008.

[5] S. Wang, M. Khafizov, X. Tu, M. Zheng, and T. D. Krauss, "Multiple exciton generation in single-walled carbon nanotubes," Nano Letters, vol. 10, no. 7, pp. 2381-2386, 2010.

[6] M. B. Tzolov, T. F. Kuo, D. A. Straus, A. Yin, and J. Xu, "Carbon nanotube-silicon heterojunction arrays and infrared photocurrent responses," Journal of Physical Chemistry C, vol. 111, no. 15, pp. 5800-5804, 2007.

[7] K. W. C. Lai, N. Xi, C. K. M. Fung, H. Chen, and T. J. Tarn, "Engineering the band gap of carbon nanotube for infrared sensors," Applied Physics Letters, vol. 95, no. 22, Article ID 221107, 2009.

[8] P. G. Collins, M. S. Arnold, and P. Avouris, "Engineering carbon nanotubes and nanotube circuits using electrical breakdown," Science, vol. 292, no. 5517, pp. 706-709, 2001.

[9] E. R. Fossum, "CMOS image sensors: electronic camera-ona-chip," IEEE Transactions on Electron Devices, vol. 44, no. 10, pp. 1689-1698, 1997.

[10] Y. Ishihara and K. Tanigaki, "A high sensitivity IL-CCD image sensor with monolithic resin lens array," in Proceedings of the IEEE International Electron Devices Meeting Technical Diges, pp. 497-500, 1983.

[11] W. A. Murray and W. L. Barnes, "Plasmonic materials," Advanced Materials, vol. 19, no. 22, pp. 3771-3782, 2007.

[12] J. A. Schuller, E. S. Barnard, W. Cai, Y. C. Jun, J. S. White, and M. L. Brongersma, "Plasmonics for extreme light concentration and manipulation," Nature Materials, vol. 9, no. 3, pp. 193-204, 2010.

[13] P. Bharadwaj, B. Deutsch, and L. Novotny, "Optical antennas," Advances in Optics and Photonics, vol. 1, no. 3, pp. 438-483, 2009.

[14] H. A. Atwater and A. Polman, "Plasmonics for improved photovoltaic devices," Nature Materials, vol. 9, no. 3, pp. 205-213, 2010.

[15] Z. Fan, D. J. Ruebusch, A. A. Rathore et al., "Challenges and prospects of nanopillar-based solar cells," Nano Research, vol. 2, no. 11, pp. 829-843, 2009.

[16] D. M. Schaadt, B. Feng, and E. T. Yu, "Enhanced semiconductor optical absorption via surface plasmon excitation in metal nanoparticles," Applied Physics Letters, vol. 86, no. 6, Article ID 063106, pp. 1-3, 2005.

[17] Z. Yu, G. Veronis, S. Fan, and M. L. Brongersma, "Design of midinfrared photodetectors enhanced by surface plasmons on grating structures," Applied Physics Letters, vol. 89, no. 15, Article ID 151116, 2006.

[18] M. W. Knight, H. Sobhani, P. Nordlander, and N. J. Halas, "Photodetection with active optical antennas," Science, vol. 332, no. 6030, pp. 702-704, 2011.

[19] N. M. Gabor, Z. Zhong, K. Bosnick, J. Park, and P. L. McEuen, "Extremely efficient multiple electron-hole pair generation in carbon nanotube photodiodes," Science, vol. 325, no. 5946, pp. 1367-1371, 2009.

[20] O. Hayden, R. Agarwal, and C. M. Lieber, "Nanoscale avalanche photodiodes for highly sensitive and spatially resolved photon detection," Nature Materials, vol. 5, no. 5, pp. 352-356, 2006.
[21] C. Soci, A. Zhang, B. Xiang et al., "ZnO nanowire UV photodetectors with high internal gain," Nano Letters, vol. 7, no. 4, pp. 1003-1009, 2007.

[22] H. Chen, N. Xi, K. W. C. Lai, C. K. M. Fung, and R. Yans, "Development of infrared detectors using single carbonnanotube-based field-effect transistors," IEEE Transactions on Nanotechnology, vol. 9, no. 5, pp. 582-589, 2010.

[23] P. J. Schuck, D. P. Fromm, A. Sundaramurthy, G. S. Kino, and W. E. Moerner, "Improving the mismatch between light and nanoscale objects with gold bowtie nanoantennas," Physical Review Letters, vol. 94, no. 1, Article ID 017402, 2005.

[24] D. P. Fromm, A. Sundaramurthy, P. James Schuck, G. Kino, and W. E. Moerner, "Gap-dependent optical coupling of single "bowtie" nanoantennas resonant in the visible," Nano Letters, vol. 4, no. 5, pp. 957-961, 2004.

[25] C. K. M. Fung, N. Xi, B. Shanker, and K. W. C. Lai, "Nanoresonant signal boosters for carbon nanotube based infrared detectors," Nanotechnology, vol. 20, no. 18, Article ID 185201, 2009.

[26] F. Léonard and J. Tersoff, "Novel length scales in nanotube devices," Physical Review Letters, vol. 83, no. 24, pp. 51745177, 1999.

[27] H. Chen, N. Xi, K. W. Lai, C. K. Fung, L. Chen, and J. Lou, "Analysis and design of carbon nanotube based field effect transistors for nano infrared sensors," in Proceedings of the IEEE Nanotechnology Materials and Devices Conference (NMDC'10), pp. 164-168, 2010.

[28] A. Sundaramurthy, K. B. Crozier, G. S. Kino, D. P. Fromm, P. J. Schuck, and W. E. Moerner, "Field enhancement and gapdependent resonance in a system of two opposing tip-to-tip Au nanotriangles," Physical Review B, vol. 72, no. 16, pp. 1-6, 2005.

[29] K. B. Crozier, A. Sundaramurthy, G. S. Kino, and C. F. Quate, "Optical antennas: resonators for local field enhancement," Journal of Applied Physics, vol. 94, no. 7, pp. 4632-4642, 2003.

[30] E. Betzig, J. K. Trautman, T. D. Harris, J. S. Weiner, and R. L. Kostelak, "Breaking the diffraction barrier: optical microscopy on a nanometric scale," Science, vol. 251, no. 5000, pp. 14681470, 1991.

[31] R. A. Pala, J. White, E. Barnard, J. Liu, and M. L. Brongersma, "Design of plasmonic thin-film solar cells with broadband absorption enhancements," Advanced Materials, vol. 21, no. 34, pp. 3504-3509, 2009.

[32] W. Ding, R. Bachelot, S. Kostcheev, P. Royer, and R. Espiau De Lamaestre, "Surface plasmon resonances in silver Bowtie nanoantennas with varied bow angles," Journal of Applied Physics, vol. 108, no. 12, Article ID 124314, 2010.

[33] K. L. Shuford, M. A. Ratner, and G. C. Schatz, "Multipolar excitation in triangular nanoprisms," Journal of Chemical Physics, vol. 123, no. 11, pp. 1-9, 2005.

[34] K. W. C. Lai, N. Xi, C. K. M. Fung et al., "Automated nanomanufacturing system to assemble carbon nanotube based devices," International Journal of Robotics Research, vol. 28, no. 4, pp. 523-536, 2009.

[35] J. Zhang, N. Xi, L. Liu, H. Chen, K. W. C. Lai, and G. $\mathrm{Li}$, "Atomic force yields a master nanomanipulator," IEEE Nanotechnology Magazine, vol. 2, no. 2, pp. 12-17, 2008.

[36] J. Zhang, N. Xi, H. Chen, K. W. C. Lai, G. Li, and U. C. Wejinya, "Design, manufacturing, and testing of singlecarbon-nanotube-based infrared sensors," IEEE Transactions on Nanotechnology, vol. 8, no. 2, Article ID 4663874, pp. 245$251,2009$. 

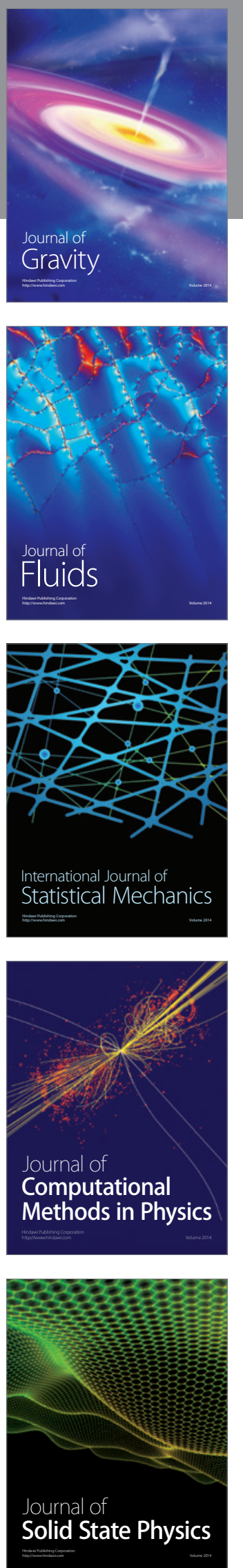

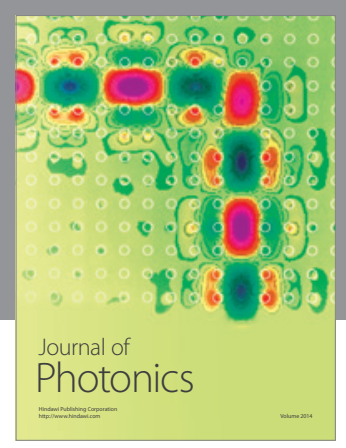

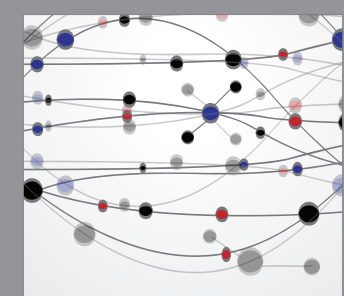

The Scientific World Journal
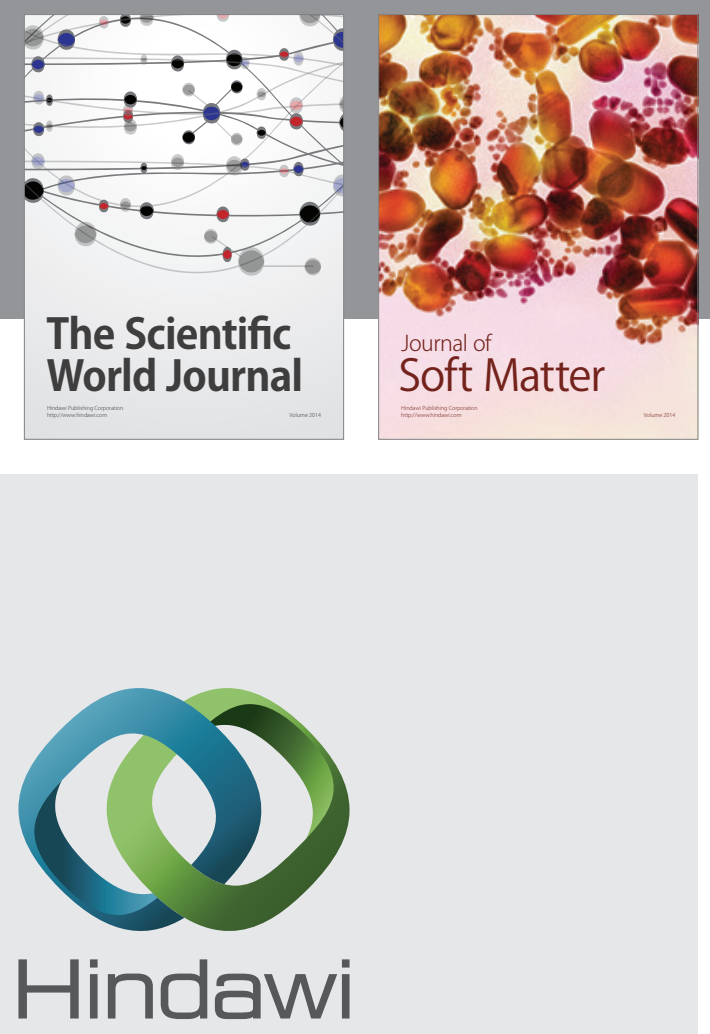

Submit your manuscripts at

http://www.hindawi.com
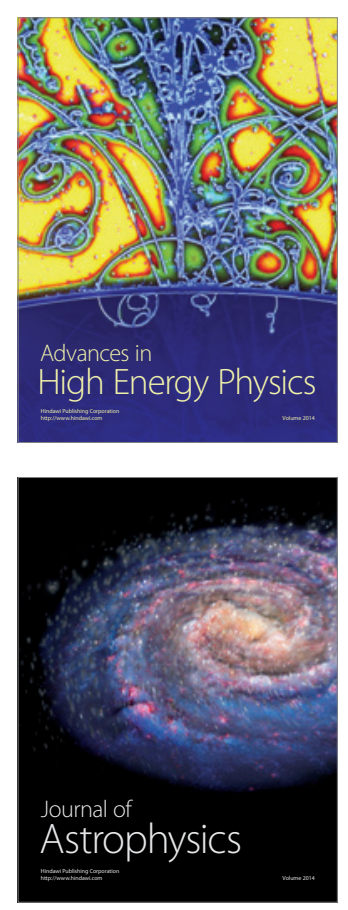
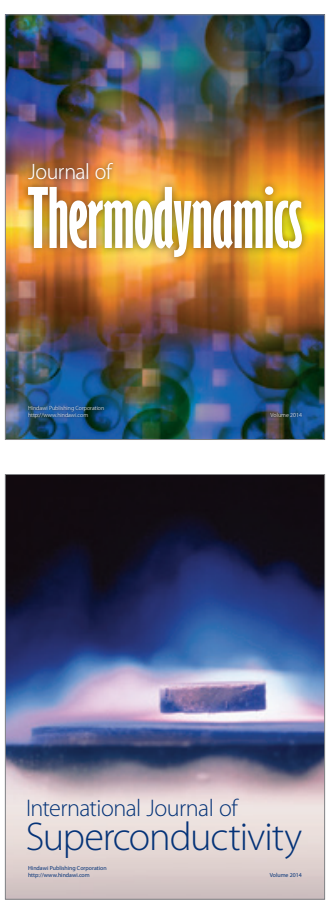
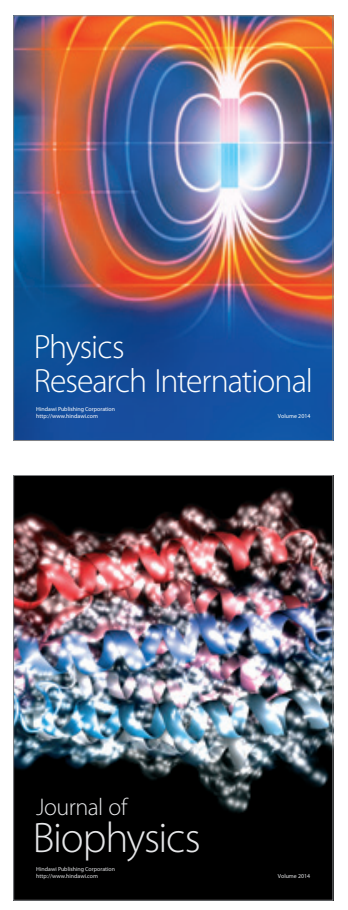
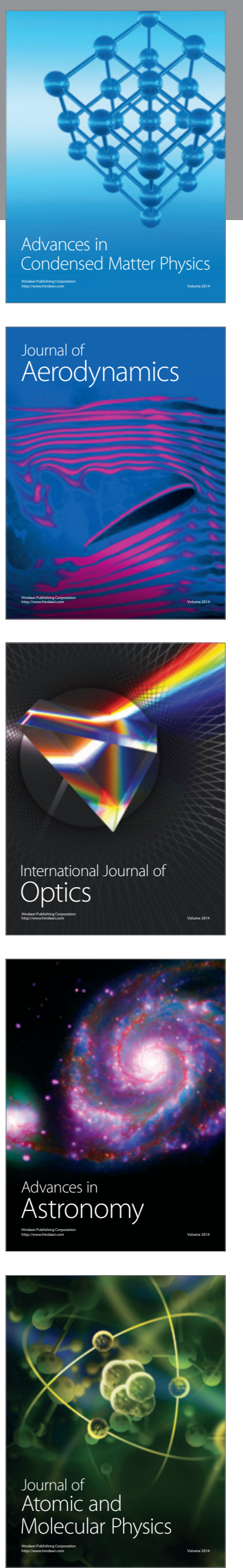